\title{
Incorporating Spiritual Healing into Modern Medicine
}

\author{
Rashmi Aggarwal ${ }^{1}$ Hemant Aggarwal ${ }^{2}$ \\ ${ }^{1}$ Department of Thyroid and Endocrine Research, \\ Institute of Nuclear Medicine and Allied Sciences (INMAS), \\ Timarpur, Delhi, India \\ 2B. K. Hospital, Faridabad, Haryana, India
}

J Soc Health Diab 2019;7:1-2

The onset of 21st century has seen a surge in spiritual awareness among people across the globe. Spirituality plays an important role in holistic management of patients suffering from all kinds of illnesses, especially terminal illnesses like cancer. It has been observed that patients whose spiritual needs have been truly addressed by their treating health care professional show a better quality of life and also experience greater well-being especially those suffering from malignancy. ${ }^{1}$

Spiritual dimension of patient: Alternative medical care in Eastern countries especially in India and neighboring countries like China, Tibet, Sri Lanka, Burma, and Nepal are usually based on spirituality and religiosity. However, in Western countries, the medical care usually revolves around physical well-being of the patient, where the spiritual aspect is not being taken care of. But lately, the Western world has also realized the usefulness of spirituality in maintaining human health and well-being. About 75\% of American medical universities, as part of their curriculum, have included spiritual care in their medical courses. In Germany, several medical schools offer course in spiritual care. ${ }^{2}$

Health care professional should devote some time listening to the spiritual beliefs of their patients.

So including spiritual training in medical curriculum would empower the young budding doctors to provide holistic care to the patients. Health has physical, mental, and social dimension as well. While imparting care to our patients, we must consider their spiritual dimensions as well. So if we ignore the spiritual dimensions, we may not be able to heal the person completely.

\section{Spiritual Interventions Undertaken by Health Care Providers}

Health care providers should respect the religious and spiritual beliefs of their patients. Just as we take medical, family, past, and menstrual history, we must also register the spiritual history of our patients. Taking the spiritual history ensures deep doctor-patient relationship, which also has a positive impact on overall health and well-being of the patient. The various spiritual interventions which can be undertaken by the health care providers are as follows:

\section{- Meditation. \\ - Counseling. \\ - Forgiveness.}

These spiritual interventions enhance both the mental as well as the physical health of the patient and help them cope up with stress and anxiety. A meta-analysis of 350 studies discovered that spirituality inclined people are physically healthier, mentally stronger, and need fewer health care services. ${ }^{3}$

\section{Spiritual History Taking}

A consensus panel of American College of Physicians has suggested four simple questions that health care providers should ask their patients ${ }^{4}$ :

1. Is spirituality important to you?

2. Has spirituality been important to you throughout your life time?

3. Do you have someone to talk to, about spiritual matters?

4. Would you like to explore spiritual matter with someone?

There is ample evidence in medical literature today that suggests that cognitive therapies may be more effective, if they take into account the spiritual belief of the patient.

D'Souza and Rodrigo conducted a randomized control trial and concluded that spiritually augmented cognitive behavior therapy was more effective in those patients who rated spirituality as important or very important. ${ }^{5}$ Recognizing and addressing the spiritual concern of the patients is crucial for imparting holistic health care to our patients. Incorporating spiritual care in our medical curriculum
Address for correspondence Rashmi Aggarwal, MD, Department of Thyroid and Endocrine Research, Institute of Nuclear medicine and Allied Sciences. (INMAS), Brig SK Majumdar Marg, Timarpur, Delhi-54, India (e-mail: drarashmi@yahoo.co.in).
DOI https://doi.org/

10.1055/s-0039-1692329

ISSN 2321-0656.
(C)2019 Novo Nordisk Education Foundation
License terms

$($ () (1) $\Theta \circledast$ 
is the need of the hour to improve overall health and well-being of the patients.

To provide holistic health care, patients' spiritual dimensions should never be neglected. Spiritual health care when incorporated in our treatment regimens, promote early recovery, prevent relapse, and improve overall quality of life. ${ }^{6}$

\section{Conclusion}

The spiritual dimension of the patient should always be acknowledged and the spiritual need of the patient should be addressed by the health care provider. The best way to acknowledge the spiritual dimension of the patient is by taking spiritual history of the patient. Exploring the spiritual dimension of the patient leads to the formation of a strong doctor-patient relationship bond which in itself leads to positive outcome in terms of achieving overall health and well-being of our patients. Therefore, spiritual care should be incorporated in medical curriculum so that young doctors are well versed in imparting spiritual care to their patients.

\section{Conflict of Interest}

None declared.

\section{References}

1 Delgado-Guay MO, Hui D, Parsons HA, et al. Spirituality, religiosity, and spiritual pain in advanced cancer patients. J Pain Symptom Manage 2011;41(6):986-994

2 Puchalski CM, Blatt B, Kogan M, Butler A. Spirituality and health: the development of a field. Acad Med 2014;89(1):10-16

3 Hummer RA, Rogers RG, Nam CB, Ellison CG. Religious involvement and U.S. adult mortality. Demography 1999;36(2): 273-285

4 Lo B, Quill T, Tulsky J. Discussing Palliative Care with patients. Ann Intern Med 1999;130:744-749

5 D'Souza RF, Rodrigo A. Spiritually augmented cognitive behavioural therapy. Australas Psychiatry 2004;12(2):148-152

6 Chidarikire S. Spirituality; the neglected dimension of holistic mental health care. Adv Ment Health 2012;10(3):298-302 